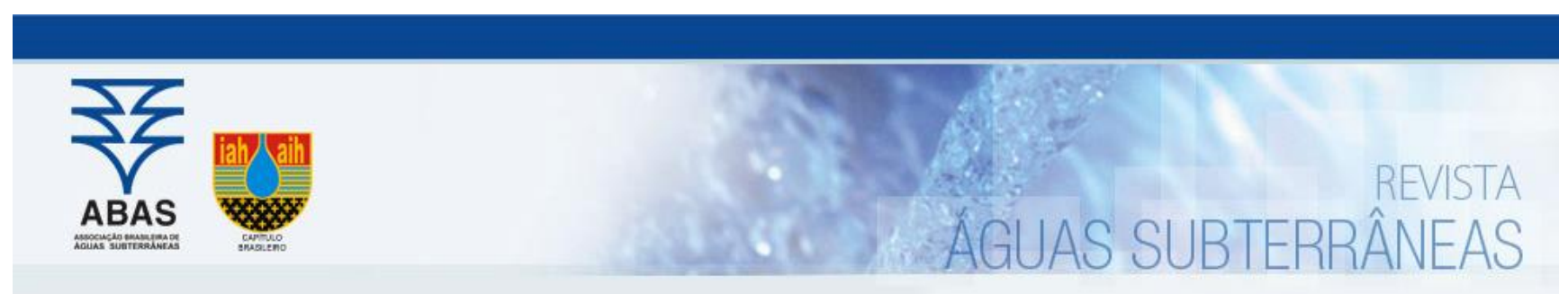

Artigos

\title{
Análise do sistema de captação de água com enfoque no rebaixamento do nível dinâmico dos poços da cidade de São Carlos-SP
}

\author{
Water supply system evaluation with approach in the lowering of the \\ dynamic groundwater levels of the wells of the São Carlos city - Brazil
}

Thais Sarinho Beham ${ }^{1}$; Maristela Oliveira Santos ${ }^{1} \bowtie$

1 Universidade de São Paulo (USP), São Paulo, SP

thabeham@gmail.com, mari@icmc.usp.br

Palavras-chave:

Abastecimento Público.

Sustentabilidade.

Rebaixamento do nível dinâmico de poços.

Otimização linear inteira mista.

\section{Keywords:}

Water Supply System.

Sustainability.

Dynamic groundwater level lowering.

Mixed-Integer Programming.

Revisado por pares.

Recebido em: 24/06/2019.

Aprovado em: 10/02/2020.
Resumo

No estado de São Paulo, nos últimos anos, há uma crescente preferência ao uso da água proveniente de poços para o abastecimento público. Isso se deve à diminuição dos custos de bombeamento da água subterrânea e à deterioração da qualidade das águas dos rios. Todavia, esta decisão é geralmente realizada apenas com base em fatores econômicos. Para estudar a situação do sistema de abastecimento da área urbana do município de São Carlos, foi desenvolvido um modelo matemático de otimização linear inteira que considerou a captação e o rebaixamento do nível dinâmico dos poços e a captação dos rios em um período de 30 anos. Para testar os modelos, foram gerados dois cenários considerando diferentes custos para o rebaixamento do nível dinâmico. Vale ressaltar que este trabalho apenas considerou o rebaixamento devido a superexplotação e não ponderou outros fatores como a influência entre os poços e as condições de recarga do aquífero. As soluções obtidas indicam uma projeção otimizada para a proporção de captação de água subterrânea e a superficial. Os resultados apresentam a melhor solução encontrada em termos de minimizar os impactos ambientais pois representam a configuração de operação que otimiza a resiliência do nível dinâmico dos poços e cumpre as restrições impostas que visam garantir a sustentabilidade do sistema.

Abstract

It is notable the growing preference in the use of groundwater into the water supply system of São Paulo State- Brazil in the last years. This occurs because of pumping cost are getting cheaper and it is happening the deterioration of the quality of the rivers' water. Nevertheless, this decision is usually made only in base in economic factors. To study the system, it was developed a mixed integer linear programming model which considers the lowering of the wells' dynamic groundwater levels and the pumping of rivers and wells in a period of 30 years. To test the model, were made scenarios that consider diverse situations of costs to the lowering of the dynamic water table from the wells. The solutions simulate how the system would work in the future in terms of how many units of wells would be activated and how the lowering would happen. The results reveal the best solution found to minimize the environmental impacts by meaning the best arrangement of the system operation to optimize the resilience of the groundwater levels and adapt to the constraints which claim to guarantee the sustainability of the system.

DOI: http:/dx.doi.org/10.14295/ras.v34i1.29565

\section{INTRODUÇÃO}

A insustentabilidade do uso da água é um problema crescente e grave observado em algumas regiões do Brasil, em especial no estado de São Paulo. Neste local, além do maior aglomerado populacional do país, o potencial hídrico é baixo para suprir a demanda (LOMBA, 2010). Exemplo disso é o estresse hídrico que a região metropolitana de São Paulo sofreu em 2014 no Sistema Cantareira.

O demasiado aumento e dependência que se tem gerado dos recursos subterrâneos é ilustrado pelo fato de $75 \%$ dos municípios do estado de São Paulo serem total ou parcialmente abastecidos por água subterrânea (DIAS, 2016). Costa (2010) alega que a outorga de uso dessas águas é realizada sem que haja critérios coerentes ou estudos do volume explotável adequado. A exploração é, em geral, realizada até o limite. 0 autor justifica o uso pela diminuição dos custos de bombeamento da água e má qualidade das águas superficiais.

Alguns países enfrentam situações drásticas atualmente, como o aquífero de Ogallala, no Arizona, nos Estados Unidos, que pode desaparecer pois já perdeu o equivalente a 18 vezes o volume do rio Colorado devido seu uso intenso na irrigação da agricultura (APRILE, 2016). Na Indonésia, a exploração desenfreada dos aquíferos fez o mar avançar cerca de 15 quilômetros para o interior (APRILE, 2016). Em Bancoc, capital da 
Tailândia, o solo em alguns lugares afunda cerca de $14 \mathrm{~cm}$ por ano devido a captação exagerada da água subterrânea que faz o solo ceder (APRILE, 2016). Como exemplo mais local tem-se que a cidade de Ribeirão Preto-SP, à aproximadamente $100 \mathrm{~km}$ de São Carlos-SP, apresenta taxas de rebaixamento do nível dinâmico do aquífero de 1 metro por ano (OLIVEIRA, 2016).

Com isso, esse trabalho visa analisar o sistema de captação de água de São Carlos que vem progressivamente criando grande dependência da captação da água subterrânea. Em 2014, 54\% da captação era realizado por poços (SAAE, 2016). A escolha de suprir o abastecimento (superficial ou subterrâneo) é embasada atualmente prioritariamente em questões econômicas e, desta maneira, visa-se analisar como a captação irá interferir nos níveis freáticos do aquífero e estimar seu

\section{DEFINIÇÃO DO PROBLEMA E MODELAGEM MATEMÁTICA}

O problema analisado consiste em formular uma função a ser minimizada buscando a melhor solução em termos da proporção de água subterrânea e superficial e diferenciando também qual a vazão em cada poço com a finalidade de conciliar um menor rebaixamento. Cabe ressaltar que existem metodologias para analisar rebaixamentos excessivos como as descritas por Feitosa e Costa (1998), entretanto este trabalho propôs uma abordagem diferente para o problema.

\subsection{Estimativa do valor do rebaixamento}

O rebaixamento foi projetado em cenários futuros ao se analisar seu comportamento em períodos anteriores. Estes dados são provenientes do trabalho de Perroni e Wendland (2008). Eles foram obtidos com base nas séries históricas disponiveis no período de 1984 a 2004, e nos ensaios de bombeamento realizados após a construção dos poços. Detalhes acerca da maneira que foi realizada essa medição, sobre o tempo que os poços estavam desligados anteriormente, não foram divulgados no estudo.

Dessa forma, cria-se uma relação entre o quanto cada poço teve o seu nível dinâmico diminuído segundo a série histórica e sua quantidade de água captada por ano. Assim, foi possível extrapolar essa relação para estimar seu desempenho no futuro. Portanto, neste trabalho é denominado de rebaixamento a diferenciação das medições nos poços de captação que foram estimadas com base em dados anteriores. Deste modo, o valor analisado é devido a fenômenos químicos e físicos somados ao rebaixamento do nível do aquífero.

Para ilustrar, segue o exemplo das informações do poço de abastecimento público número 2, denominado Antônio Fischer dos Santos (PERRONI e WENDLAND, 2008):

- Rebaixamento: $31 \mathrm{~m}$

- Idade: 34 anos

- Vazão: $48,5 \mathrm{~m}^{3} / \mathrm{h}$

- Profundidade: $130 \mathrm{~m}$ comportamento ao longo dos anos pela geração de dois cenários através da proposição de um modelo de otimização. Um cenário com custo de rebaixamento 1000 vezes maior que o primeiro.

Desta forma, pretende-se analisar a configuração do sistema de captação de água de São Carlos-SP através de um modelo matemático a ser otimizado e prever seu comportamento em um período de 30 anos. Para tal, estudam-se as proporções de água subterrânea e superficial e o rebaixamento do nível dinâmico da água dos poços considerando custos diferenciados em cada tipo de captação e no rebaixamento. É importante salientar que este trabalho apenas considerou o rebaixamento devido a superexplotação e não ponderou outros fatores como a influência entre os poços e as condições de recarga do aquífero.

Como este poço teve seu nível dinâmico diminuído em 31 m em um período de 34 anos, pode-se aproximar que houve um rebaixamento de 0,912 m ao ano, em média, conforme equação (1). Denomina-se esse parâmetro de Rm.

$$
\begin{array}{r}
R m=\text { rebaixamento total } / \text { idade } \\
=31 / 34 \\
=0,912 \mathrm{~m} / \text { ano }
\end{array}
$$

Pela sua vazão e com um funcionamento de 20 horas por dia, tem-se que o volume total de água retirado por ano (Vol) é de $354050 \mathrm{~m}^{3}$ (equação (2)).

$$
\begin{aligned}
\operatorname{Vol}=\operatorname{Vaz} \tilde{\mathrm{A}}\left[\mathrm{m}^{3} / \mathrm{h}\right] & \cdot(20 \mathrm{~h} \cdot 365 \text { dias }) \\
= & 48,5 \cdot 20 \cdot 365 \\
& =354050\left(\mathrm{~m}^{3} . \text { ano }\right)
\end{aligned}
$$

Assim, relacionando as expressões (1) e (2), pode-se gerar uma relação entre o volume captado e o rebaixamento obtido, denominada de 'R'. Neste caso, o valor de R é de 2,576 m/ $10^{6} \mathrm{~m}^{3}$. ano, conforme equação (3)

$$
R=\frac{R m}{V o l}=\frac{0,912}{354050}=2,576 \frac{m}{10^{6} m^{3} \cdot \text { ano }}
$$

Isso significa que, caso se retirem $1.000 .000 \mathrm{~m}^{3}$ desse poço durante um ano, ele deterá um rebaixamento estimado em 2,58 metros. Este releva uma estimativa simplificada, pois não considera outros fatores como o raio de influência dos outros poços, cone de rebaixamento, princípio da superposição, heterogeneidades no meio poroso, períodos de recarga, e se a vazão e as horas de funcionamento permaneceram constantes em todo o período da idade do poço.

Dessa forma, aplicando para os demais poços, tem-se os valores apresentados na Tabela 1:

A localização destes poços pode ser observada na Figura 1: 
Tabela 1 - Valores dos parâmetros Rm, Vol e R

\begin{tabular}{llll}
\hline Poço & $\mathrm{Rm}(\mathrm{m} / \mathrm{ano})$ & $\mathrm{Vol}\left(\mathrm{m}^{3} / \mathrm{ano}\right)$ & $\mathrm{R}\left[\frac{m}{10^{6} \mathrm{~m}^{3} \cdot \text { ano }}\right]$ \\
\hline 2 & 0,9120 & 354050 & 2,576 \\
5 & 0,0430 & 289080 & 0,149 \\
8 & 0,3000 & 131400 & 2,283 \\
9 & 0,1180 & 679630 & 0,174 \\
11 & 1,0000 & 693500 & 1,442 \\
12 & 0,7330 & 954110 & 0,768 \\
14 & 0,9170 & 128480 & 7,137 \\
16 & 1,7270 & 1142450 & 1,512 \\
17 & 3,8890 & 1252680 & 3,105 \\
19 & 1,0000 & 162060 & 6,171 \\
20 & 3,4290 & 2092910 & 1,638 \\
21 & 1,4000 & 912500 & 1,534 \\
22 & 0,6000 & 483990 & 1,240 \\
23 & 0,7500 & 1049740 & 0,714 \\
24 & 0,6670 & 2080500 & 0,321 \\
26 & 4,0000 & 954110 & 4,192 \\
28 & 4,0000 & 1330060 & 3,007 \\
\hline
\end{tabular}

Fonte: Adaptado de Perroni e Wendland (2008)

Figura 1 - Localização dos poços

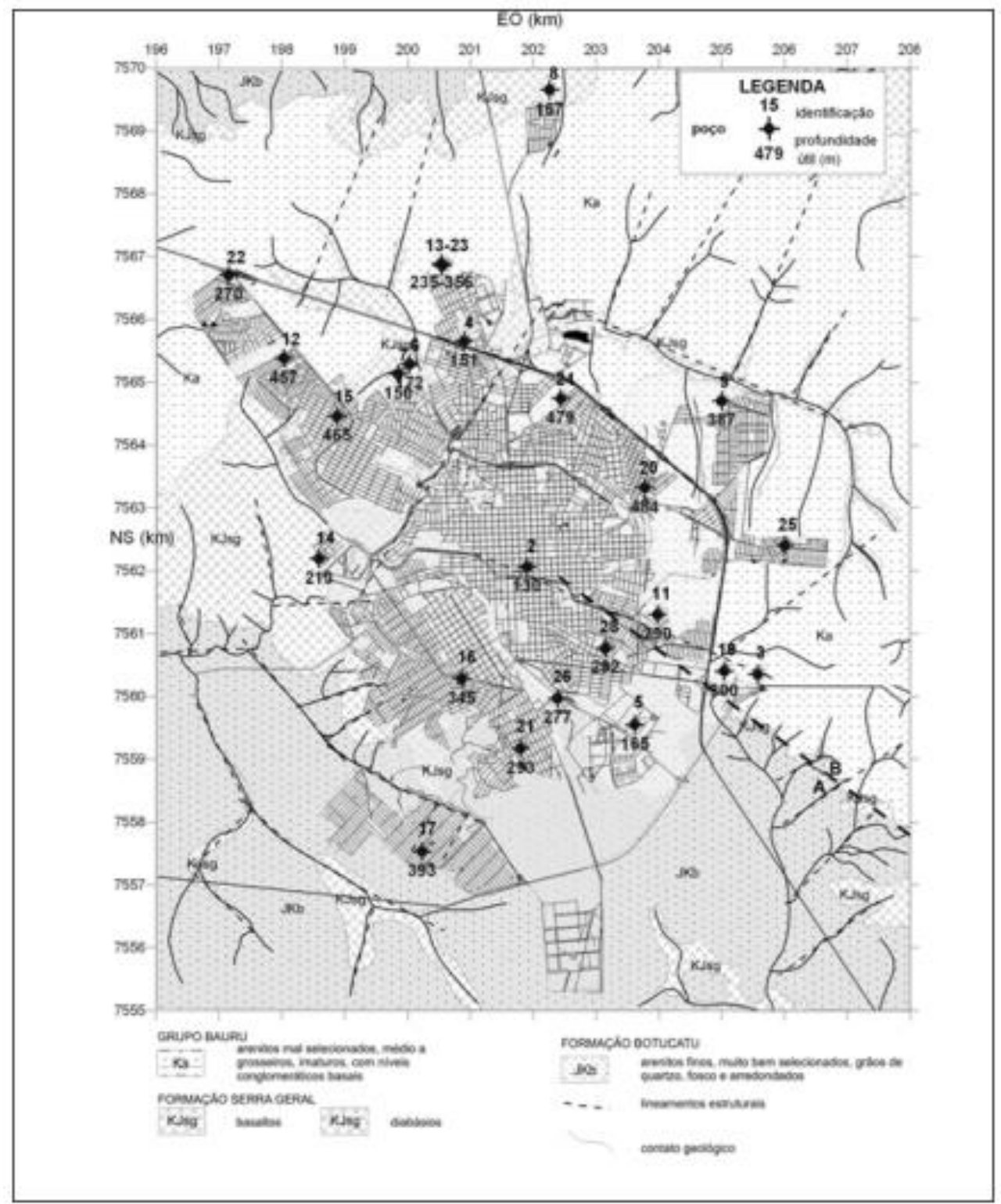

Fonte: Extraído de Perroni e Wendland (2008) 
Adicionalmente, é importante apontar que há poços dentro do município que não possuem outorga. Entretanto, para este trabalho focou-se apenas nos poços cadastrados para abastecimento público.

\subsection{Modelo matemático}

As decisões principais consistem em decidir a proporção entre a captação subterrânea de cada poço $i$ em cada período de tempo $t$ e a captação superficial de cada rio $j$ no período $t$ com objetivo de atender as demandas a cada ano. Cada período $t$ representa um ano dentro de um horizonte de tempo de $T$ anos. A demanda de cada ano té determinística e será basea- da no consumo per capita, crescimento da população e perdas no sistema de abastecimento do município em questão.

Para estudar os impactos da captação de água em cada rio $j$, estabelece-se faixas para captação, as quais correspondem a uma fração do total disponível. Cada faixa $k$ apresenta um custo diferenciado para o volume captado no rio $j$. Isso significa que o custo de captação varia conforme a quantidade retirada. Caso seja bombeado um volume muito grande, este valor estará contido em uma faixa que representa um custo mais elevado em comparação à um volume menor.

Os seguintes índices são utilizados:

\begin{tabular}{l|l}
\hline \multicolumn{1}{l|}{ Índices } & \\
\hline $\mathrm{N}$ & número total de poços \\
\hline $\mathrm{J}$ & número total de rios \\
\hline $\mathrm{T}$ & número total de períodos \\
\hline $\mathrm{K}$ & número total de faixas para os rios \\
\hline $\mathrm{M}$ & número total de faixas para os poços \\
\hline$i \in\{1, \ldots, \mathrm{N}\}$ & índice dos poços \\
\hline$j \in\{1, \ldots, \mathrm{J}\}$ & índice dos rios \\
\hline$t \in\{1, \ldots, \mathrm{T}\}$ & índice dos períodos \\
\hline$k \in\{1, \ldots, \mathrm{K}\}$ & índice das faixas dos rios \\
\hline$m \in\{1, \ldots, \mathrm{M}\}$ & índice das faixas dos poços \\
\hline
\end{tabular}

Assim como as seguintes variáveis são utilizadas:

\begin{tabular}{|c|c|}
\hline Variávei & \\
\hline $\mathrm{Xp}_{\mathrm{it}}$ & Volume de água retirado do poço $i$ no período $t$ \\
\hline $\mathrm{XFp_{i,t,m }}$ & Volume de água retirado do poço $i$ no período $t$ na faixa $m$ \\
\hline $\mathrm{Xr}_{\mathrm{jt}}$ & Volume de água retirado do rio $j$ no período $t$ \\
\hline $\mathrm{XFr} \mathrm{j}_{\mathrm{j}, \mathrm{k}, \mathrm{k}}$ & Volume de água retirado do rio $j$ no período $t$ na faixa $k$ \\
\hline $\mathrm{YFp} \mathrm{Fi}_{\mathrm{i}, \mathrm{m}}$ & $\begin{array}{l}1 \text { se ocorre captação de água no poço i na faixa } m \text { durante o período } t \text { e } \\
0 \text {, caso contrário }\end{array}$ \\
\hline$Y F r_{j, t, k}$ & $\begin{array}{l}1 \text { se ocorre captação de água no rio } j \text { na faixa } k \text { durante o período } t \text { e } 0 \text {, } \\
\text { caso contrário }\end{array}$ \\
\hline Rebit & Rebaixamento no poço $i$ no período $t$. \\
\hline
\end{tabular}

As seguintes constantes são utilizadas:

\begin{tabular}{l|l}
\hline \multicolumn{1}{c|}{ Constantes } & \\
\hline $\mathrm{Cr}_{\mathrm{j}, \mathrm{k}, \mathrm{t}}$ & Custo de retirada da água do rio $j$ na faixa $k$ no período $t$ \\
\hline $\mathrm{Cp}_{\mathrm{i}, \mathrm{m}, \mathrm{t}}$ & Custo de retirada da água do poço $i$ na faixa $m$ no período $t$ \\
\hline $\mathrm{Creb}_{\mathrm{i}}$ & Custo do rebaixamento do poço $i$ \\
\hline $\mathrm{Q} i$ & Vazão inicial do poço $i$ \\
\hline $\mathrm{PP}_{\mathrm{it}}$ & Perda de volume de água na captação do poço i no período $t$ \\
\hline $\mathrm{PR}_{\mathrm{j} t}$ & Perda de volume de água na captação do rio $j$ no período $t$ \\
\hline$V_{\mathrm{rjt}}$ & Vazão do rio $j$ no período $t$ \\
\hline $\mathrm{PC} j k\left(\mathrm{~F}_{\mathrm{j}}\right)$ & Faixa de captação utilizada no rio $j$ (fração de captação) \\
\hline $\mathrm{PPCi}, \mathrm{m}$ & Faixa de captação utilizada no poço $i$ na faixa $m$ \\
\hline $\mathrm{Ri}$ & Rebaixamento inicial do poço $i$ \\
\hline
\end{tabular}

Deste modo, a formulação matemática do modelo pode ser crito pelas expressões (4) - (16). descrita como um problema de minimização dos custos des- 


$$
\min \sum_{k=1}^{K} \sum_{j=1}^{J} \sum_{t=1}^{T} c r_{j, k, t} \cdot X F r_{j, k, t}+\sum_{m=1}^{M} \sum_{i=1}^{N} \sum_{t=1}^{T} c p_{i, m, t} \cdot X F p_{i, m, t}+\sum_{t=1}^{T} \sum_{i=1}^{N} \operatorname{Creb}_{i} \cdot \operatorname{Reb}_{i, t}
$$

SUJEITO À:

$$
\begin{aligned}
& \sum_{i=1}^{N}\left(1-P P_{i, t}\right) \cdot X p_{i, t}+\sum_{j=1}^{J}\left(1-P R_{j, t}\right) \cdot X r_{j, t} \geq D_{t} \\
& X r_{j t}=\sum_{k=1}^{K} X F r_{j, t, k} \\
& P C_{j, k-1} \cdot V r_{j, t} \cdot F_{j} \cdot Y F r_{j, t, k} \leq X F r_{j, k, t} \leq P C_{j, k} \cdot V r_{j, t} \cdot F_{j} \cdot Y F r_{j, t, k} \\
& \sum_{k=1}^{K} Y F r_{j, t, k} \leq 1 \\
& \sum_{i=1}^{I} X p_{i, t} \leq 0,05 \cdot \text { chuva }_{t} \\
& X p_{j t}=\sum_{m=1}^{M} X F p_{i, t, k} \\
& P P C_{i, m-1} \cdot Q_{i} \cdot Y F p_{i, t, m} \leq X F p_{i, t, m} \leq P P C_{i, m} \cdot Q_{i} \cdot Y F p_{i, t, m} \\
& \sum_{m=1}^{M} Y F p_{i, t, m} \leq 1 \\
& R e b_{i, t}=R e b_{i, t-1}+R_{i} \cdot X p_{i, t} \\
& \forall \mathrm{J}, \mathrm{T} \text {; } \\
& X F r_{j, t, k}, X F p_{i, t, m} \geq 0 \\
& \forall \mathrm{I}, \mathrm{J}, \mathrm{K}, \mathrm{M}, \mathrm{T} \text {; } \\
& Y F p_{i, t, m} \in\{0,1\}, Y F r_{j, t, k} \in\{0,1\} \\
& \forall \mathrm{I}, \mathrm{J}, \mathrm{K}, \mathrm{M}, \mathrm{T} \text {; } \\
& X r_{j, t}, R e b_{i, t}, X p_{i, t} \geq 0
\end{aligned}
$$

Sendo então, a função objetivo (4) representa a minimização dos custos que são formados por três parcelas. A primeira parcela é referente a captação superficial, a segunda relacionada à captação dos poços e a última, aos custos de rebaixamento dos poços. As restrições (5) definem que o volume total captado das duas fontes deve ser suficiente para suprir a demanda da população no período $t$. Considera-se que existe uma perda para cada poço $i$ no período $t$ e também existe uma perda na água captada do rio $j$ no período $t$. Essa perda é relativa à rede de distribuição de água. As restrições (6) determinam a quantidade de água captada no período $t$ no rio $j \mathrm{e}$ essa depende da faixa utilizada e juntamente com as restrições (7) e (8) estabelecem o volume de água captado no rio $j$ bem como a faixa que poderá ser utilizada para a captação de água. 
As restrições (9) limitam a captação total subterrânea em 5\% do volume de água de chuvas no período $t$. Esta porcentagem é definida com base no conceito de reserva ativa do aquífero, o valor representa uma estimativa do volume que estaria disponível para captação e distribuição para abastecimento (SANTOS, 2009). Para se calcular o volume de chuva das restrições (9) utiliza-se dos dados de pluviometria e da área de atuação da bacia da mancha urbana da cidade que é a subbacia hidrográfica do Rio Monjolinho, parte da bacia do TietêJacaré. As restrições (10) determinam a quantidade de água captada no período $t$ no poço $i$ e esta quantidade depende da faixa utilizada. As restrições (11) e (12) estabelecem o volume de água captado no poço $i$ bem como a faixa que poderá ser utilizada para a captação de água. As restrições (13) referemse ao cálculo do rebaixamento de cada poço devido à captação de água total no período t. Ademais, esse é um valor acumulativo, sendo que o valor do ano anterior é somado para se obter o resultado do rebaixamento no tempo presente, pois conforme descrito anteriormente, trata-se de uma simplificação do problema. Por fim, as restrições (14), (15) e (16) definem o domínio das variáveis.

\section{RESULTADOS E DISCUSSÕES}

O modelo matemático foi implementado em linguagem $\mathrm{C}++$ utilizando-se a ferramenta Concert Technology do solver comercial ILOG CPLEX(TM) 12.1. Dois cenários foram executados (descritos nos itens 3.1 e 3.2) durante um tempo máximo limitado em 15 minutos e o tempo necessário para o solver encontrar a solução ótima para todos os cenários foi inferior a 8 segundos. A configuração do CPLEX foi alterada para parar com um desvio (GAP) relativo de 10-1. Este desvio é calculado por GAP = 100(FO-LB)/FO, onde FO é o valor da função objetivo do modelo e LB é o limitante inferior, ambos valores são obtidos pelo CPLEX.

Nas simulações, utilizados os seguintes valores de parâme- tros:

$\mathrm{N}: 17$ (número de poços)

- $\quad J=2$ (número de rios)

- $\mathrm{T}=30$ (período de anos)

- $\mathrm{K}=2$ (número de faixas do rio)

- $M=2$ (número de faixas do poço)

Os valores propícios para os custos foram definidos depois de vários testes realizados. Para os poços, estes tiveram seus valores proporcionais as suas constantes de rebaixamento $(\mathrm{Rm})$. Assim, as unidades com maior valor de Rm, ou seja, maior tendência a rebaixar, possuem custos maiores. A constante de rebaixamento foi multiplicada por $10^{-7}$ para definir o custo de captação no primeiro período. Cada período seguinte obteve um acréscimo de $4 \%$ que pretende representar que é mais danoso a cada ano retirar a água do rio. A segunda faixa de captação implica os mesmos custos da primeira multiplicados por 50. A constante de rebaixamento por sua vez, tiveram sus custos multiplicados por 20. A mesma proporção foi aplicada para os custos de rebaixamento de cada poço. Os rios, por sua vez, tiveram seus custos mais elevados, pois teriam que competir com o fato que as fontes subterrâneas apresentam dois tipos de penalidades: pela captação e pelo rebaixamento. Além disso, o rio com menor vazão possuiu custos mais elevados (4\%) e a cada período há um acréscimo de $2 \%$, seguindo o modelo dos poços. As perdas na rede foram consideradas nulas nos testes computacionais.

Os dados utilizados em referência à demanda foram coletados da população observada em 2018 pelo IBGE e feito uma projeção para os próximos 30 anos com um crescimento anual de $1,54 \%$, que pode ser observada no Gráfico 1. Essa porcentagem foi o valor estimado de crescimento pelo IBGE (IBGE, 2019). O consumo diário per capita foi definido por 130 Litros por habitante por dia, valor enquadrado na literatura referente à cidade do porte de São Carlos (cerca de 200.000 habitantes) (VON SPERLING, 1995).

Gráfico 1 - Consumo de água anual em Litros estimados para a cidade de São Carlos-SP

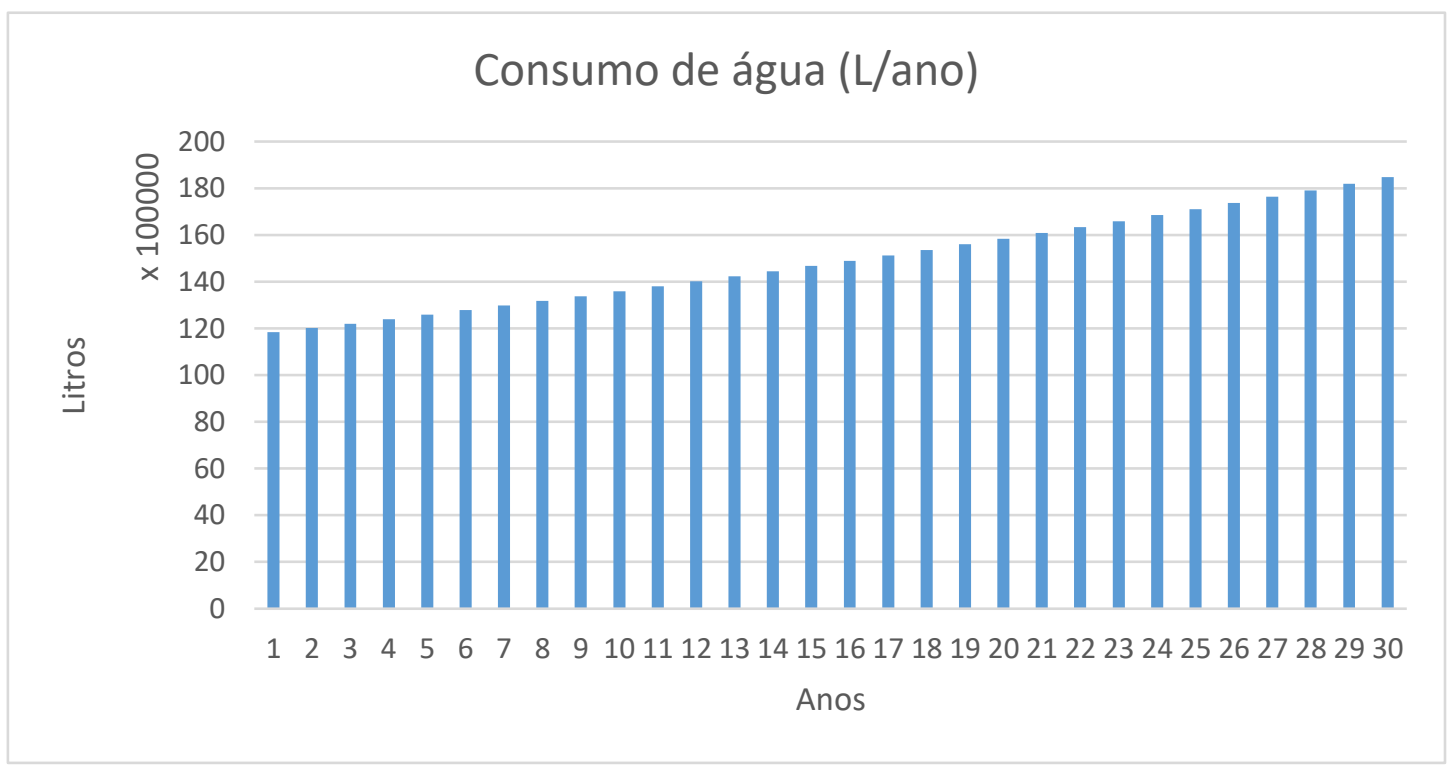


Nos dois rios que abastecem a cidade são o Rio Monjolinho e o Ribeirão do Feijão, a vazão utilizada no presente trabalho é de $2,5 \mathrm{~m}^{3} / \mathrm{s}$ para o primeiro (CAMPAGNA, 2005) e de $4 \mathrm{~m}^{3} / \mathrm{s}$ para o segundo (JUNIOR e MUAD, 2015). As taxas de precipitação foram obtidas pelo Plano Municipal de Saneamento de São Carlos de 2012 (Prefeitura de São Carlos, 2012).

Foram analisados dois cenários, o primeiro com os custos conforme definidos nesta sessão multiplicado por 10 e outro em que há um aumento de 1000 vezes em relação ao primeiro. Essa configuração pretende demonstrar o comportamento do rebaixamento do nível dinâmico estimado ao penalizar bastante sua ocorrência.

\subsection{Cenário 1: Custo de rebaixamento $=10$}

Neste cenário foi aplicado o custo de rebaixamento igual 10. 0 gráfico 2 revela a quantidade captada de cada poço separadamente no primeiro e último período, respectivamente. Observa-se que com o aumento da demanda, muitos poços que não foram usados no primeiro ano, obtiveram valores bem expressivos no último. Cabe salientar que o número de poços ativos nos períodos 1 e 30 é diferente (conforme observado no Quadro 2) e, devido a isso, é possível constatar a opção de abertura de novos poços como favorável pelos resultados obtidos nas análises. Em outras palavras, esta foi a alternativa elegida na otimização do modelo proposto.

A Tabela 2 a seguir indica quais são os poços ativos em cada período.

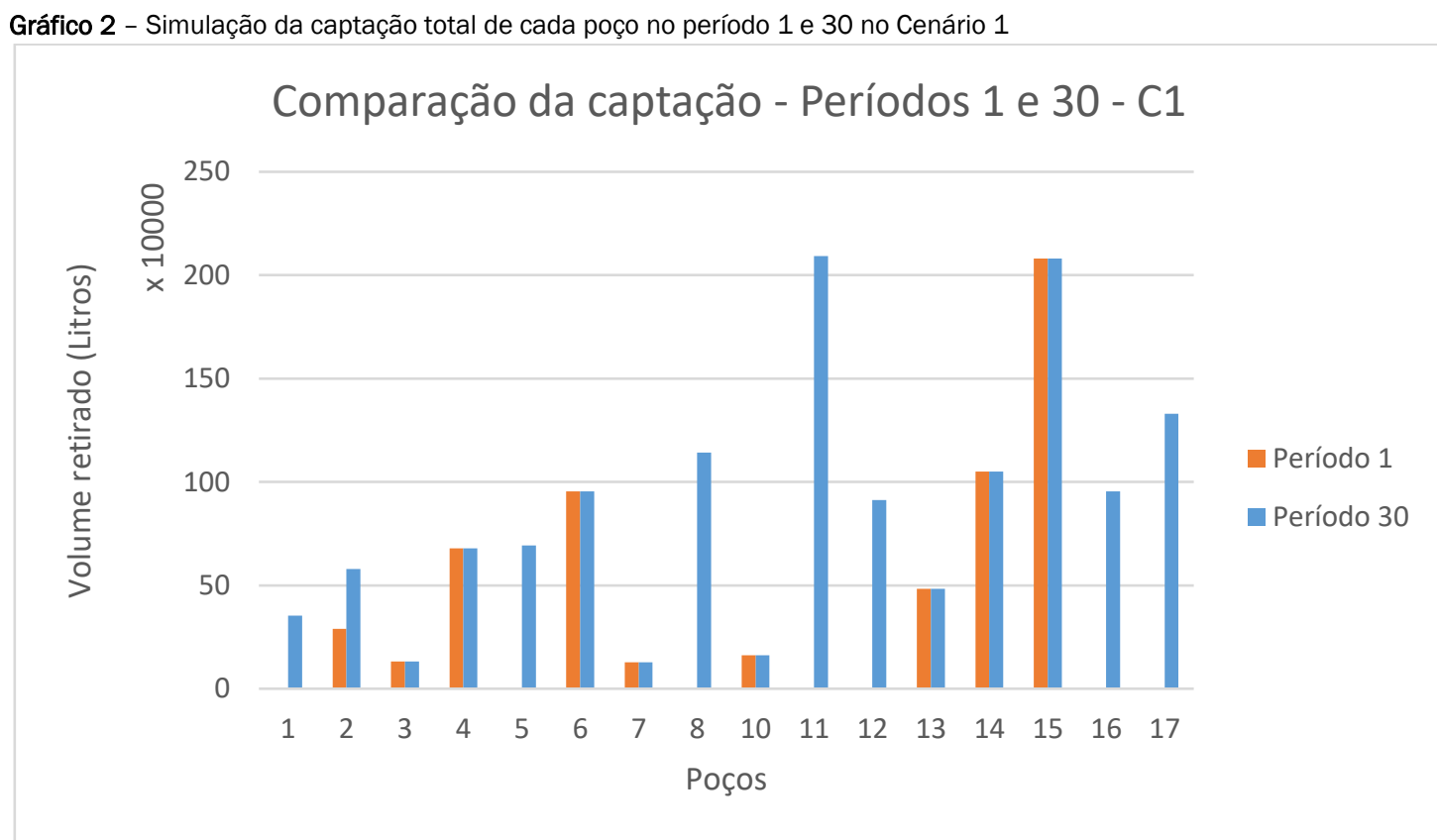

Adicionalmente, observa-se uma captação superficial oscilante com picos por volta do décimo quinto ano, conforme constatado no Gráfico 3 que expressa o volume extraído ao longo dos anos. No final da análise há valores menores que no princípio desta, significando que o aumento da demanda não gerou muito impacto nesta fonte de recurso.
Ao analisar a proporção entre a captação subterrânea e superficial ao longo dos anos, conforme Gráfico 4, constatou-se que essa se manteve similar (próxima a 50\%) durante os primeiros 5 anos e em seguida foi crescendo o percentual de uso do poço até atingir valores acima de $70 \%$ nos últimos 4 anos. 
Tabela 2 - Poços ativos em cada período no Cenário 1

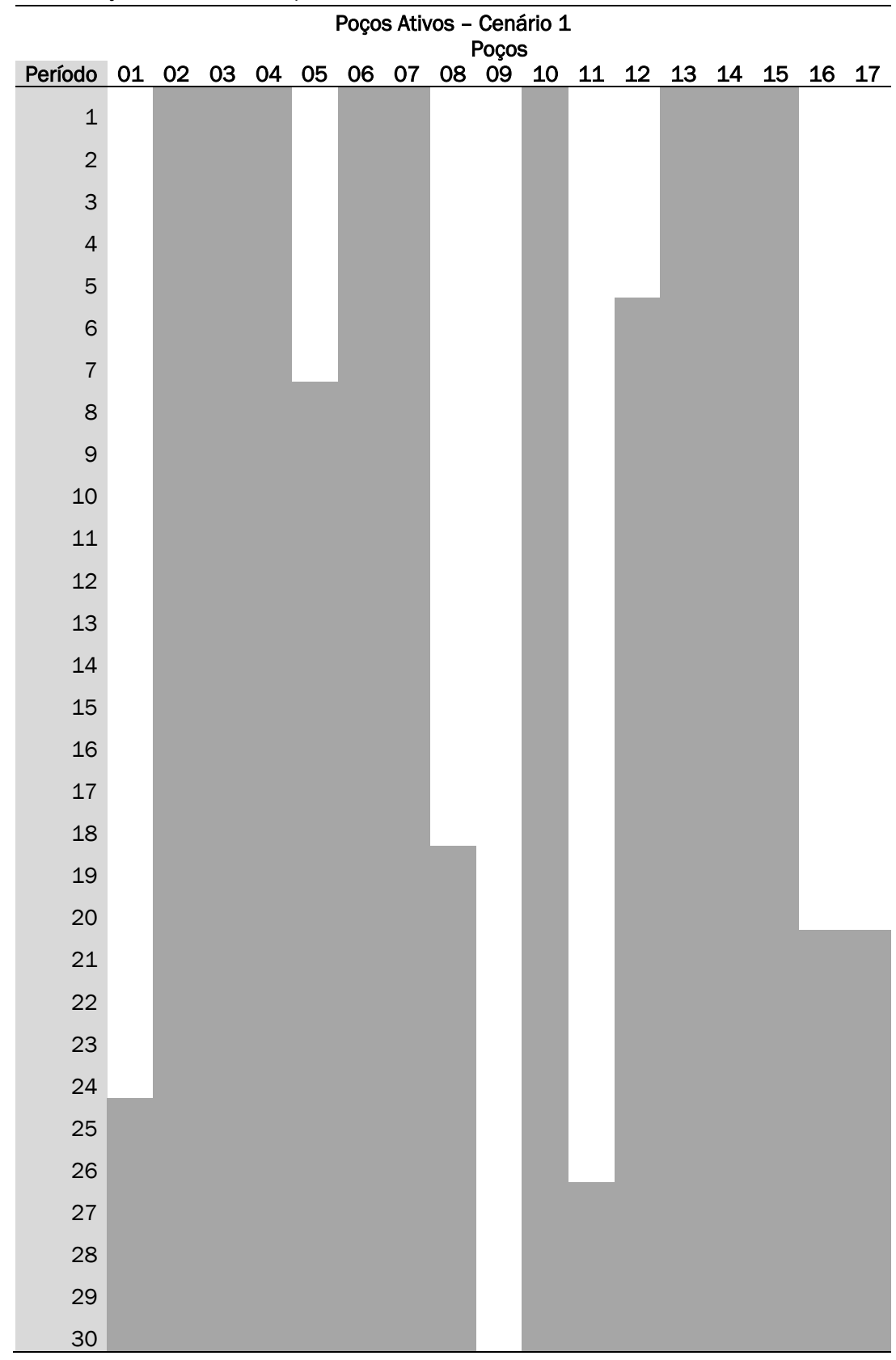

Gráfico 3 - Captação superficial através dos anos no Cenário 1

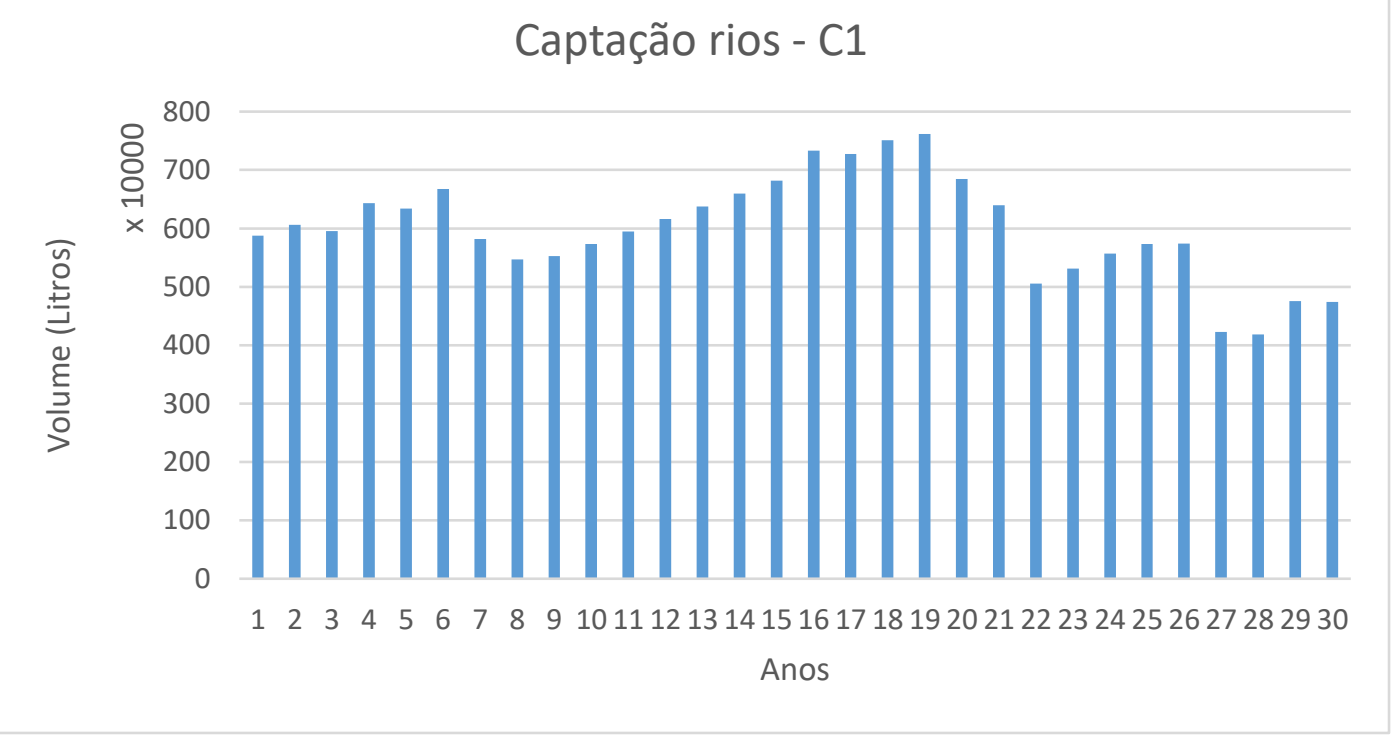


Gráfico 4 - Percentual de captação subterrânea e superficial anual do Cenário 1

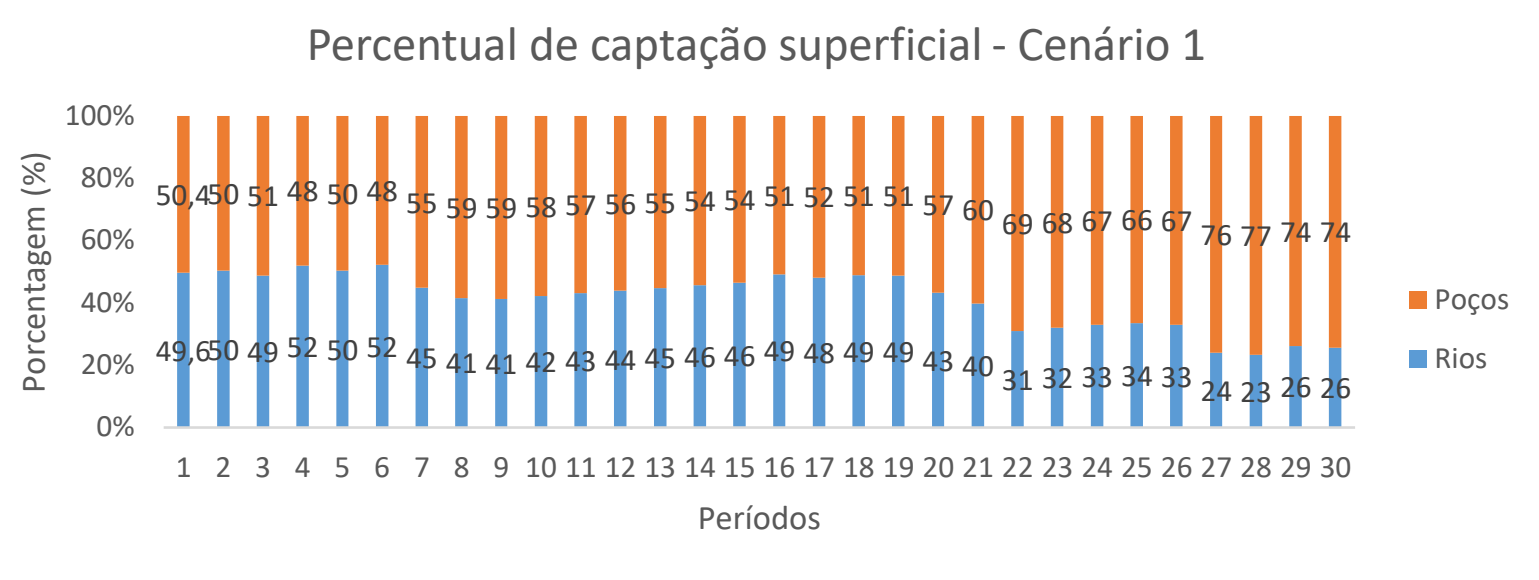

0 rebaixamento foi limitado a 35 metros durante as simulações. Desta forma, evitou-se que algumas unidades tivessem resultado muito discrepante em relação aos demais. 0 Gráfico
5 indica como se portou esse rebaixamento no primeiro $(t=1)$ e último período $(t=30)$. Também é apresentado o rebaixamento inicial, indicado por " $\mathrm{t}=0$ ".

Gráfico 5 - Rebaixamento do nível dinâmico dos poços no Cenário 1

\section{Rebaixamento do nível dinâmico - C1}

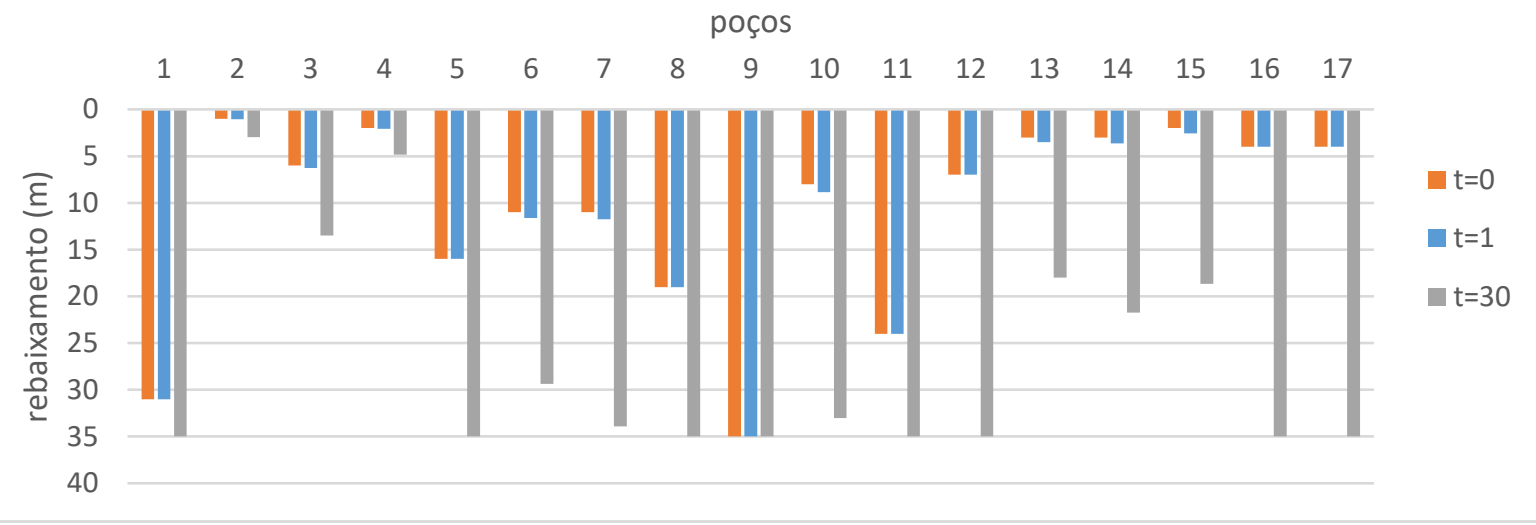

Conforme explicado na Seção 2.2 - Modelo matemático, na captação tanto superficial como subterrânea o custo é atribuído em faixas conforme o total retirado. Além disso, cada poço apresenta diferentes valores de faixa que são baseadas na sua constante de rebaixamento, ou seja, na sua tendência de diminuição do nível dinâmico. Assim, neste cenário, apenas o poço 2 atingiu o limiar da sua faixa e teve seu custo de captação aumentado.

Seu custo de captação foi aumentado pois a quantidade de água necessária a ser bombeada para atingir a segunda faixa era baixa. Isso acontece, pois, esta fonte possui uma tendência maior em rebaixar que as outras, devido à análise histórica de seu nível d'agua. Dessa forma, apesar de ter um custo maior, o impacto no valor final da função objetivo é pequeno pois este é a somatória do produto dos custos pela quantidade de água e o volume neste caso é relativamente pequeno.

\subsection{Cenário 2: Custo do rebaixamento $=10000$}

o Cenário 2 apresenta um custo de rebaixamento de 10000. Ao se definir um custo 1000 vezes maior para o rebaixamento que a simulação anterior, pretende-se a diminuição deste impacto ambiental ao fornecer uma penalidade muito elevada para sua realização. Desta maneira, a análise resulta em uma configuração da rede com o intuito de se preservar ao máximo o nível dos poços.

O Gráfico 6 indica o volume captado nos poços no primeiro e último período. Ele revela que há uma menor discrepância entre os poços quando o mesmo período é comparado com o do cenário anterior.

Os poços 7 e 9 não estão presentes no Gráfico 6 pois não apresentaram captação durante os períodos exibidos. 0 mesmo pode ser observado na Tabela 3 que indica quais poços estão ativos em cada período. 
Gráfico 6 - Simulação da captação total de cada poço no período 1 e 30 no Cenário 2

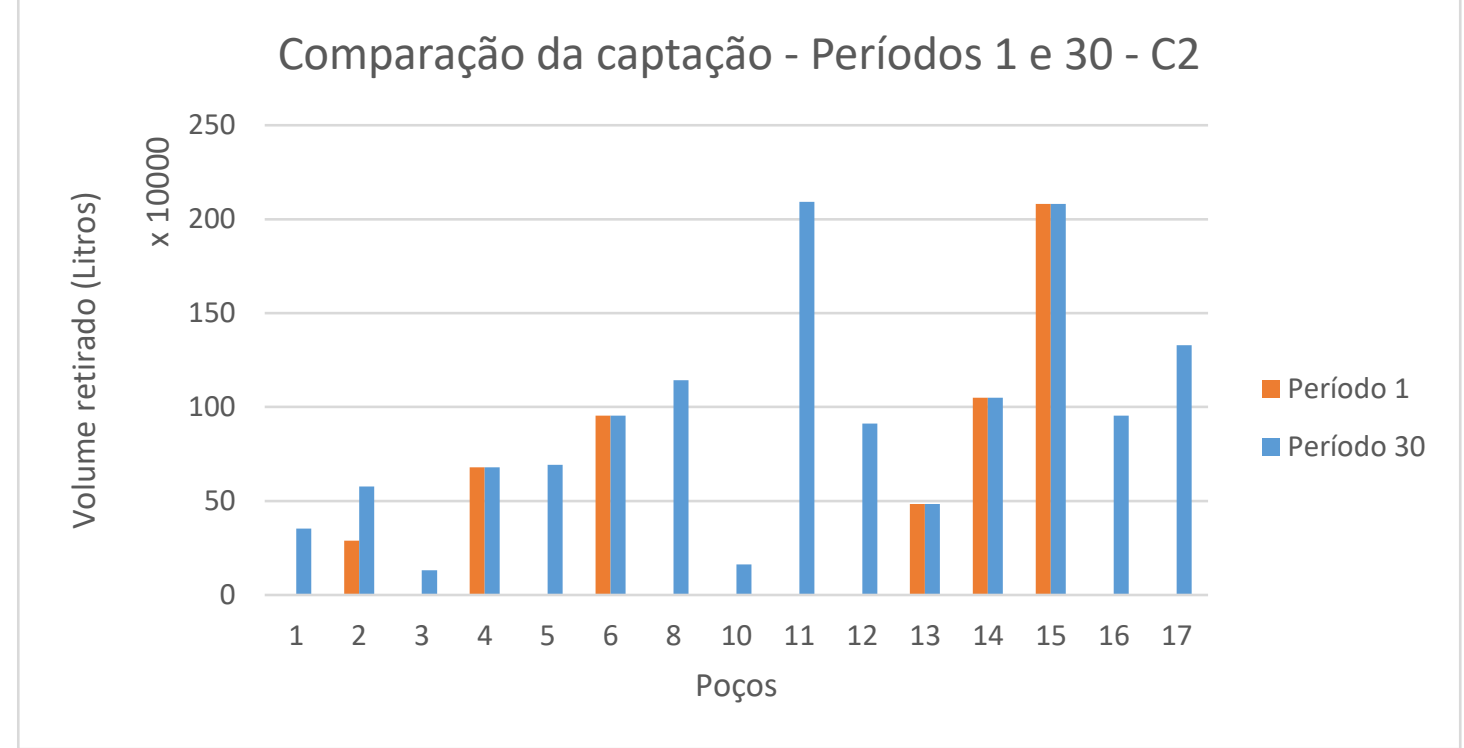

Ao apresentar já incialmente um rebaixamento muito elevado, o poço 9 não apresentou captação durante toda a análise. Os valores iniciais de rebaixamento são indicados no Gráfico 5.0 poço 7 por sua vez, foi escolhido como mais favorável a sua não utilização neste cenário.

Adicionalmente, a captação total subterrânea é menor que anteriormente, o que resulta em maior bombeamento dos rios, constatado pelos dados do Gráfico 7, que indica o total reti- rado dos rios ao longo dos anos. A captação superficial neste cenário se difere do anterior por se apresentar intensa já nos primeiros anos e ser menos oscilante.

Neste cenário a porcentagem de captação subterrânea também é crescente, vide Gráfico 8. Todavia, dentre os 30 anos analisados, 21 deles estão na faixa de $45 \%$ a $55 \%$, caracterizando como um sistema que manteve um percentual consideravelmente similar para ambas as fontes.

Gráfico 7 - Captação superficial através dos anos no Cenário 2

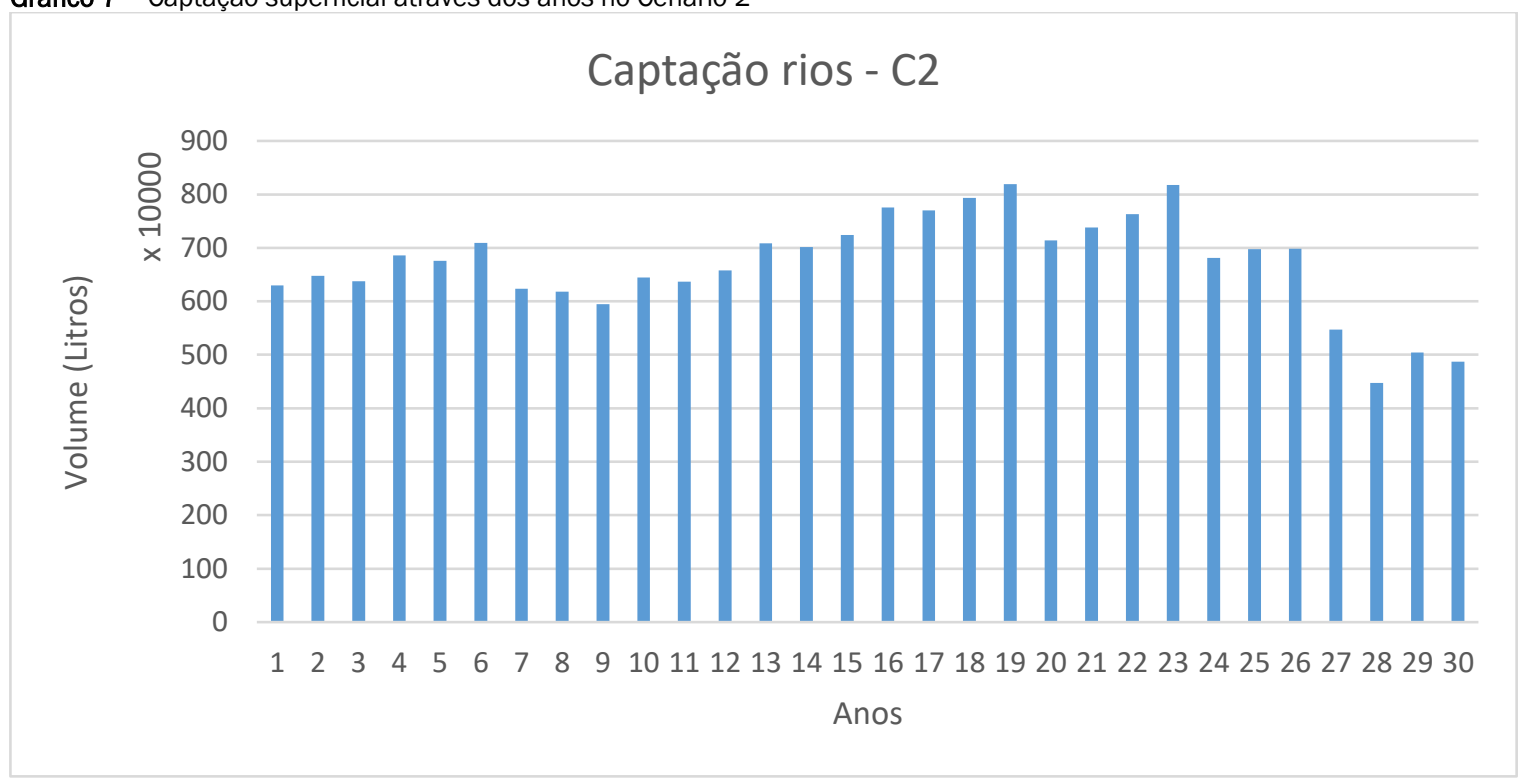




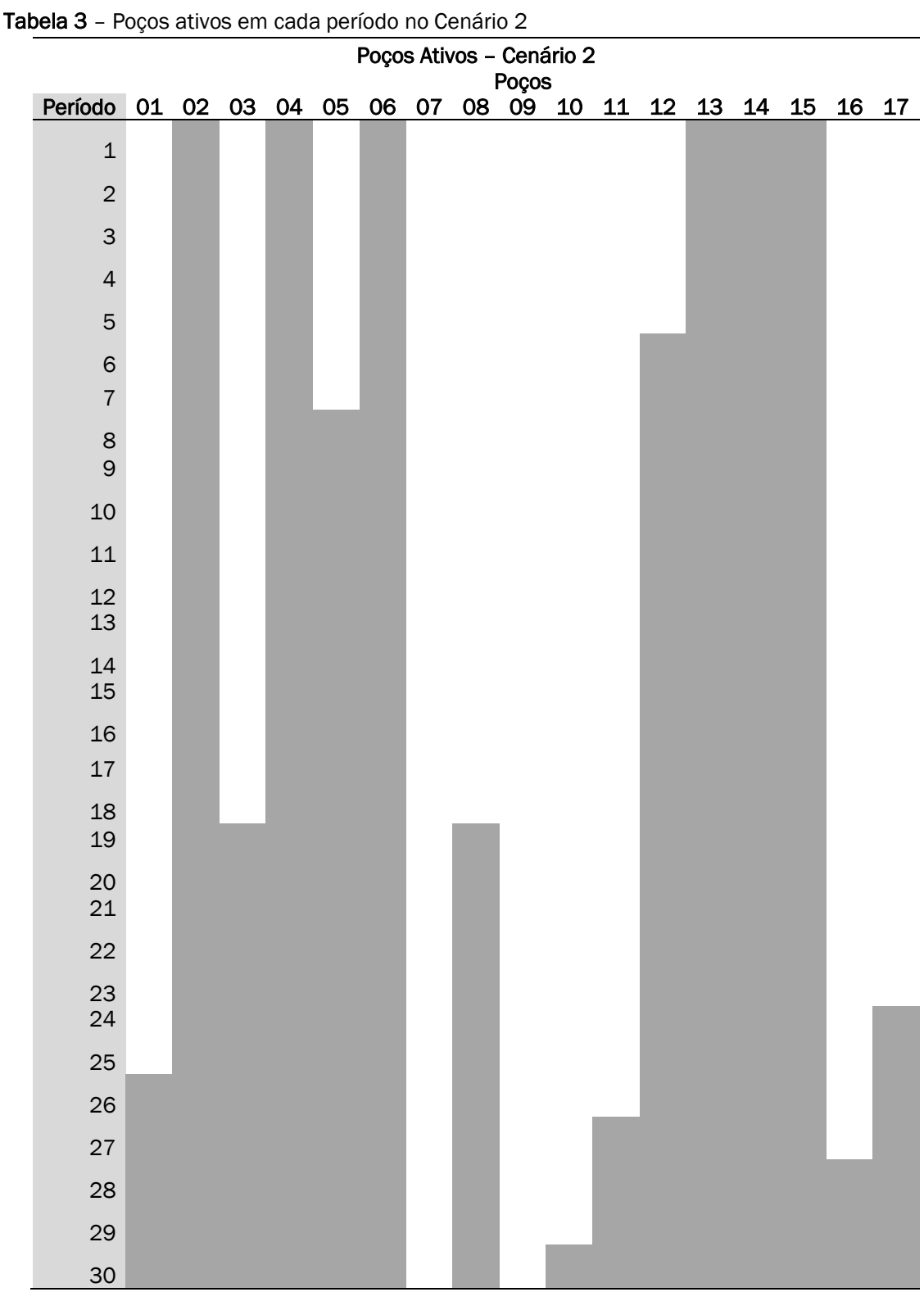

Gráfico 8 - Percentual de captação subterrânea e superficial anual do Cenário 2

Percentual de captação superficial e subterrânea - Cenário 2

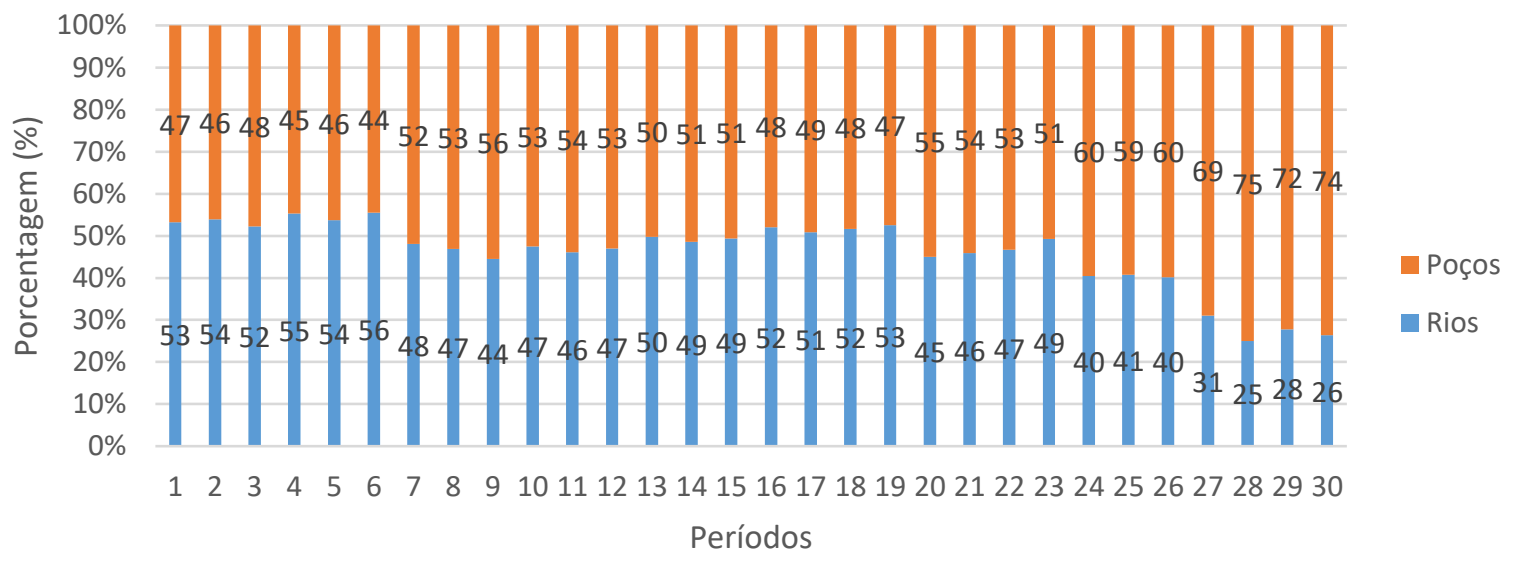


O rebaixamento, pelo Gráfico 9, apresenta-se mais favorável que no cenário anterior por apresentar valores em geral me nores e apenas 6 poços que atingiram 35 metros, enquanto na outra análise, foram 8 unidades.

Gráfico 9 - Rebaixamento do nível dinâmico dos poços no Cenário 2

\section{Rebaixamento do nível dinâmico - C2}

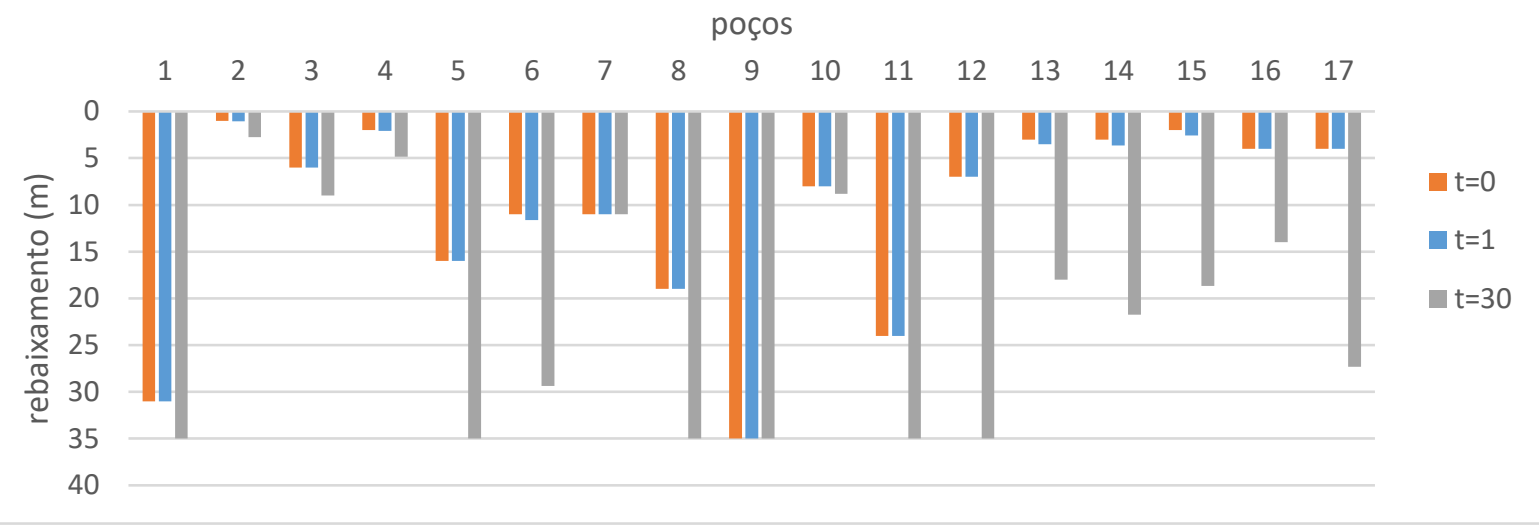

Novamente neste cenário, o poço 2 foi o único que retirou um volume de água na segunda faixa, resultando em um custo proporcional maior, assim como explicado no cenário anterior.

\section{CONCLUSÃO}

O presente trabalho estudou o comportamento do sistema de captação de água do município de São Carlos-SP. O caso considerado e suas particularidades não está presente na literatura, porém durante o trabalho foi possível criar estratégias de solução de modo a abordar o problema de maneira condizente com a situação real.

As análises dos resultados permitiram que fossem estimados os efeitos da atual tendência de aumento do uso de fontes subterrâneas e atribuir diferentes "pesos" para frear a ocorrência do rebaixamento e gerar simulações para cenários futuros.

Assim, foi possível estimar como o sistema poderia se com-

portar de duas maneiras e atendendo a demanda estipulada. Dessa forma, o estudo buscou abordar um cenário com um custo referente ao rebaixamento do nível dinâmico 1000 vezes maior que o outro. Isso proporcionou diferentes análises quanto a importância dada a um impacto ambiental. Os resultados forneceram como a operacionalização (quais poços estão ativos a cada ano) deveria ocorrer ao longo dos próximos 30 anos a fim de se minimizar ao máximo os custos e como se portaria o rebaixamento do nível dinâmico ao longo desse período.

Desta forma, as porcentagens de captação superficial e subterrânea, assim como os rebaixamentos estimados e o número de poços ativos por período, possuem a intenção de ajudar no manejo e gestão adequada deste recurso. Como a dinâmica das águas subterrâneas se caracteriza como um sistema bastante complexo, criar modelos que simulem, busquem otimizar sua operação e representem cenários futuros são de extrema importância para uma gestão mais sustentável.

\section{REFERÊNCIAS}

APRILE, J. Aquífero Guarani: águas subterrâneas também estão em risco. 2016. Disponível em: https://educacao.uol.com.br/disciplinas/geografia/aquifero-guaraniaguas-subterraneas-tambem-estao-em-risco.htm.

CAMPAGNA, A. F. Toxicidade dos sedimentos da Bacia Hidrográfica do Rio Monjolinho (São Carlos - SP): ênfase nas substâncias cobre, aldrin e heptacloro. 2005. Tese (Doutorado), 2005.

COSTA, C. W. Expansão da mancha urbana e suas consequências sobre os mananciais dor rio do Monjolinho e do ribeirão do Feijão da cidade de São Carlos, SP. 2010. Dissertação (Mestrado), 2010.

DIAS, H. Centro de pesquisa do IGc alerta para contaminação de aquíferos paulistas. 2016. Disponivel em: http://www5.usp.br/38710/ centro-de-pesquisa-do-igcalerta-para-contaminacao-de-aquiferos-nitrato/.

ESA: Engenharia Sanitária e Ambiental. Revisão e complementação do Plano Diretor de Saneamento de São Carlos. [S.I.], 2012.

FEITOSA, Fernanda AC; COSTA FILHO, Waldir Duarte. Execução de testes de bombeamento em poços tubulares: Manual prático de orientação. CPRM, 1998.

IBGE. São Carlos. 2019. Disponível em: https://cidades.ibge.gov.br/brasil/sp/sao-carlos/panorama. Acesso em: 01 fev. 2019.

JUNIOR, P. P. A.; MAUAD, F. F. Simulação dos impactos das mudanças climáticas na vazão da bacia do ribeirão do feijão - sp. Revista Brasileira de Recursos Hídricos. [S.I.: s.n.], 2015.

LOMBA, C. C. P. A disponibilidade hídrica da região metropolitana de São Paulo. In: ENCONTRO NACIONAL DOS GEÓGRAFOS, 16., 2010. [Anais...]. [S.I.: s.n.], 2010.

OLIVEIRA, A. Nivel do Aquífero Guarani baixa um metro por ano em Ribeirão Preto, SP. 2016. Disponível em: 
http://g1.globo.com/sp/ribeirao-preto-franca/noticia/2012/03/nivel-do-aquifero-guarani-baixa-um-metro-porano-em-ribeirao-preto-sp.html.

PREFEITURA SÃO CARLOS. Report, Plano Municipal de Saneamento de São Carlos. 2012.

PERRONI, J. C. A.; WENDLAND, E. Avaliação das condições de ocorrência e explotação do sistema aqüífero guarani em são carlos-sp. Águas Subterrâneas, v. 22, n. 1, 2008.
SAAE. Produção de Água e Esgoto Tratado. 2016. Disponível em: http: //www.saaesaocarlos.com.br/joomla4/index.php/dadossaneamentomenutop/ producaoaguaeesgotomenu.

SANTOS, M. M. Gerenciamento de recursos hídricos subterrâneos: uso atual e potencial do sistema aquífero guarani no estado de São Paulo-SP. 2009. Tese (Doutorado), 2009.

VON SPERLING, M. Introdução à Qualidade das Águas e ao Tratamento dos Esgotos. v.1. Belo Horizonte: UFMG, 1995. 